\title{
Differential Classification Method in Different Teaching Models of Accounting Courses Based on Naive Bayesian Classification Algorithm
}

\author{
https://doi.org/10.3991/ijet.v14i08.10395 \\ Xiuying Ou \\ Wenzhou Business College, Wenzhou, China \\ xgyue@foxmail.com
}

\begin{abstract}
Accounting is an important management discipline with strong theoretical foundation and practical operation. Due to the differences between individuals in the process of learning, the mastery of the subject is different. This requires teachers to implement differential teaching from the differences in student personality in the process of teaching. However, when teachers use the concept of difference teaching to teach, the classification of students' differences is mostly calculated by manual quantification such as records, tests, surveys, etc. This kind of measurement and qualitative method not only wastes manpower, but also has personal subjectivity, blindly relies on individual subjective judgment to judge students' advantages and interests, and has accuracy and scientificity. This requires research on students' differential classification methods. Therefore, this paper proposes a student classification method based on naive Bayesian algorithm. It constructs a classifier based on historical data, and then uses a well-structured and stable classifier to classify the actual preclassification objects, and actually applies it to the teaching of accounting courses, realizing the difference in the teaching process. Provide data support for future differential teaching research. The results show that the naive Bayesian classification algorithm can be used to analyze the difference in personality and learning of students. Presupposition and generative teaching objectives and students improve their self-awareness to better promote self-development.
\end{abstract}

Keywords - Differential classification method, naive bayesian algorithm, student differential classification method, Accounting Courses

\section{Introduction}

Accounting has a long history of development and it has a very close relationship with the economy. The course teaching of accounting majors in colleges and universities is a further improvement of the related conceptual principles, capabilities and other knowledge structures of accounting, with strong practical characteristics [1].The course teaching research on accounting is related to the investigation and research of the accounting course and various aspects involved in the practice of teaching. This research allows us to discover a series of problems that are easy to appear in the im- 
plementation of specific courses. The problem helps to have a more intuitive and rational understanding of the current teaching situation [2].

Difference teaching means that due to the innate quality of different students and the difference in acquired culture, the students' learning ability and the quality structure of students are greatly different. Quality education advocates the "studentcentered" teaching concept [3]. Accounting is a basic subject. It is necessary to implement this educational concept, respect the subjective position of students, pay attention to the individuality of students, and teach students in accordance with their aptitude. The use of differentiated teaching methods allows teachers to give full play to the guiding role in the classroom. Students' timely, appropriate and appropriate influence can promote the cultivation of students' interest and focus on discovering the potential ability of each student. However, the current problem of unequal opportunities in the teaching process is the main reason for the differences among students [4]. In the teaching process, class-based classes are used, and class-level lectures are bound to ignore the problems that some students need to solve in terms of cognitive status, attention, and knowledge construction. Obviously, if these problems are not solved, students will not be able to master the educational goals, and the differences between students will also arise. At present, most of the research on differential teaching focuses on the research of differential teaching theory, the necessity and importance of the implementation of differential teaching, the process of implementing differential teaching, and the measures to ensure the implementation of differential teaching. There are few studies on the diagnosis and diagnosis of differences.

The research in this paper is a research on differential classification methods, which is the basis for implementing differential teaching. A naive Bayesian algorithm based on classification in data mining is proposed in the research of differential classification methods. The algorithm is based on Bayes' theorem, the classification is more scientific, and the classifier has no strict limitations on the data type, and can process any type of data [5]. In addition, the classification algorithm is clear and simple in structure, and the calculation efficiency has stronger advantages than other classification algorithms. It can meet the speed requirement of large data volume training and query. When processing large amounts of data, the system overhead is small and the performance is stable. Therefore, this paper proposes a student classification method based on naive Bayesian algorithm, and applies this classification method to the accounting course, which realizes the difference in the teaching process and provide data support for future differential teaching research.

\section{Difference Teaching Mode}

\subsection{Overview of differential teaching}

The difference teaching is based on a class of students who have the objective difference in intelligence and potential, and a teaching mode that enables each student to get the most improvement on their own. Difference teaching is a kind of teaching mode based on students' natural classes. It is a teaching mode that recognizes students' 
innate intellectual differences. It is a teaching mode that respects students' individuality and specialty. It is a kind of face-to-face student intelligence. Development of a teaching model that differs in time [6].

Differentiated teaching is concentrated in the different requirements of the same course. For a classmate, each student chooses a different course. Each student has a teaching model of his own curriculum. We call it selective teaching. Middle school, especially college students, have adopted this kind of teaching. It can be said that differential teaching is primary personalized teaching [7]. Difference teaching is arranged by teachers, while selective teaching is chosen by students to varying degrees. They are different manifestations of personalized education.

The identification of difference teaching by the scholars of Hex is: Implementing the difference teaching means that the teacher changes the speed, level or type of teaching to suit the learner's needs, learning style or interest [8].

The Greg Gregory scholars identified the difference teaching as: In the difference teaching class, the teacher will actively design and implement various forms of teaching content, teaching process and teaching results according to the students' water learning interest and learning needs [9].

\subsection{Student difference analysis}

The differences in student learning are mainly due to differences in students' enthusiasm for learning. In the survey of hundreds of students, the enthusiasm of students and perseverance have the greatest impact on student learning. The enthusiasm of learning is the process of students discovering problems and solving problems independently. It is a psychological process of active exploration. Each student has a unique personality. They have the ability to think independently. Their brain cells are fully developed, the contact fibers grow rapidly, and the process of excitement and inhibition has reached equilibrium. Through the teacher's differential guidance, they are encouraged to think, strengthen their enthusiasm for learning, and strengthen the interest of students in learning.

At present, the research on the difference measurement method takes students as the main body, and refers to the difference test content pointed out by the scholars of Hex and Hua Guodong. Combining the pedagogy and educational psychology related materials, a total of 20 attributes of 5 dimensions are selected to describe one. Student's personalization [10], as shown in Figure 1.

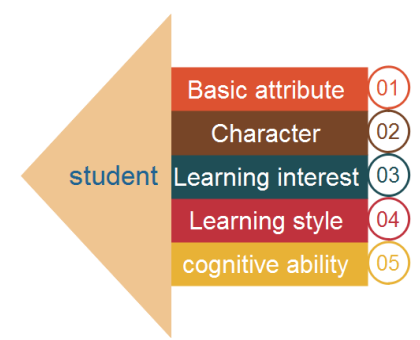

Fig. 1. Student's personalized attribute -5 dimensions 
The differences in student basic attributes are shown in Figure 2. In this paper, the basic attributes of students are considered, including gender, political appearance, and family background.

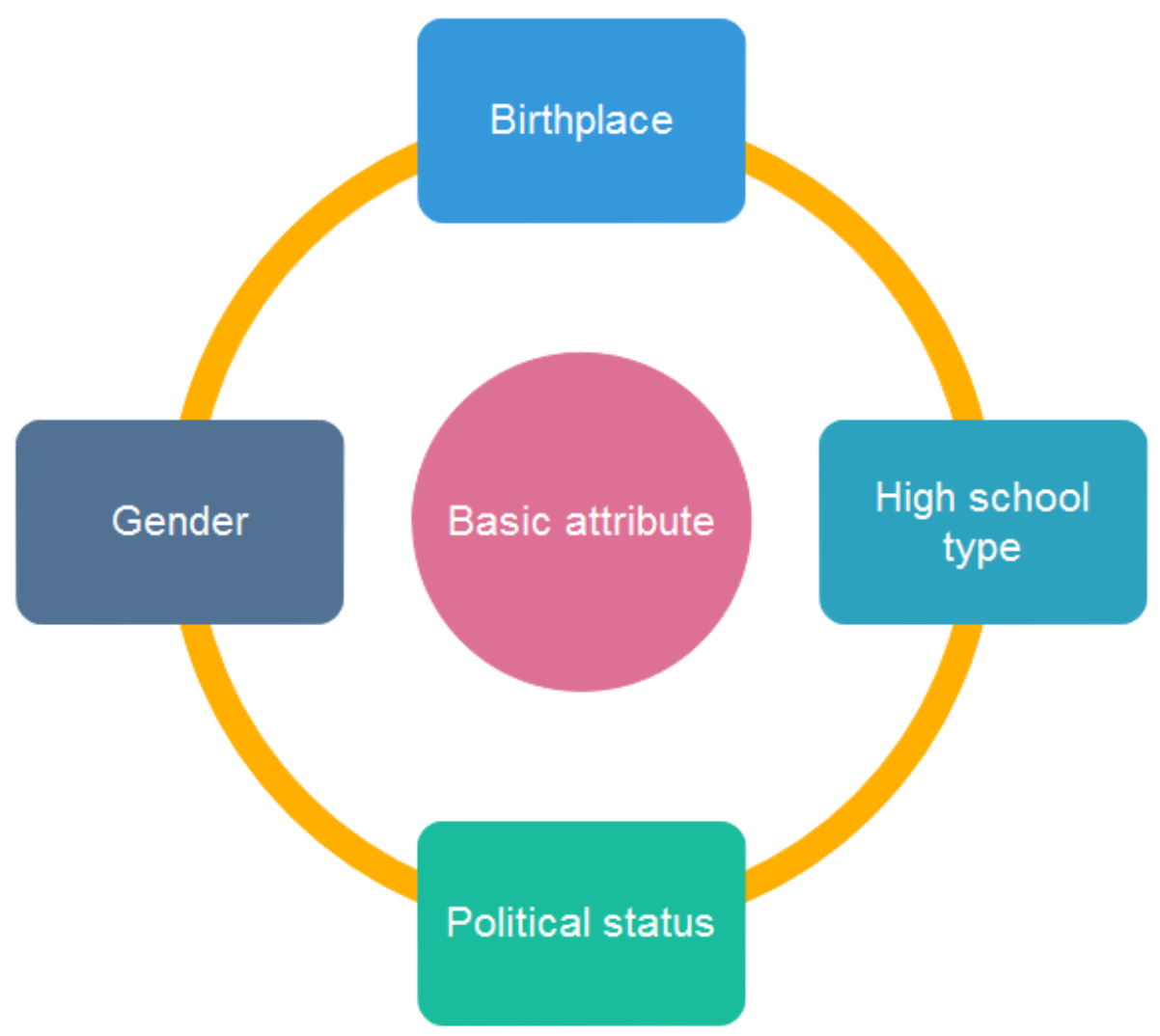

Fig. 2. Differences in basic attributes of students

The differences in student personality attributes are shown in Figure 3. The main manifestations of personality differences in the learning process are: lively, responsive, self-confident students, the classroom can actively answer questions, gain the attention of teachers and students, strengthen and enhance self-confidence; students who are impatient and reckless Can actively answer questions, but often incorrect answers, which requires teachers to distinguish the differences between students' personality, do not blindly deny students' answers, so as not to affect students' enthusiasm; slow response, silent and lonely, melancholic students, in the classroom Because the performance is not positive, they often do not participate in the classroom interaction, and are easily ignored by teachers and students. 


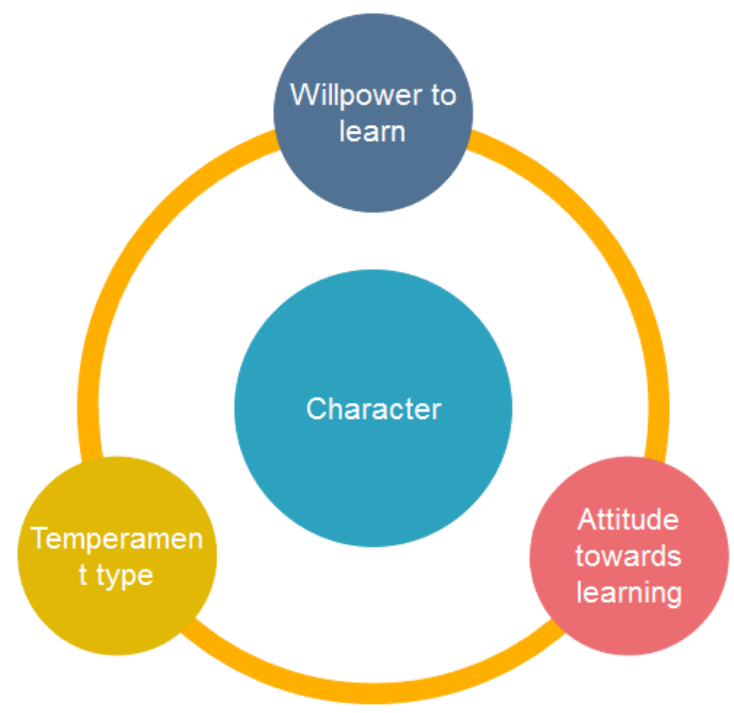

Fig. 3. Student personality attribute

The differences in student learning interest attributes are shown in Figure 4. In the study, interest is the best teacher, but the difference in interest in the curriculum is ultimately reflected in the motivation of learning, and here is the type of learning motivation proposed by Ausubel. Differences in student programs are expressed in different interests or different interests in the course. Students who are interested in the course can actively complete the teaching objectives and expand their knowledge. On the contrary, other students need teachers to repeatedly urge learning. Differences in learning interests can lead to polarization of learning outcomes, and teachers need to differentiate students' interest differences.

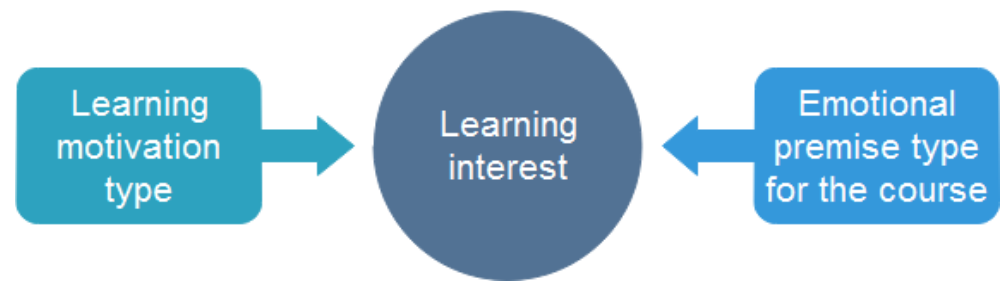

Fig. 4. Student learning interest attribute

The differences in student learning style attributes are shown in Figure 5. The differences in learning styles are mainly reflected in the differences in students' cognitive styles, learning environments, learning emotions, social factors of learning, and strategies for learning. The different manifestations of cognitive style are manifested in the process of individual perception, reasoning, understanding problems, problem solving and memory. The different needs of the learning environment and social factors are: quiet or lively learning environment, the intensity of light, independent learn- 
ing or companion learning, the best time to learn, and the stimulation of learning. The emotion of learning comes from the cultivation of students themselves and teachers and parents. The different manifestations of learning strategies are the ways in which knowledge is built in the student's learning process.

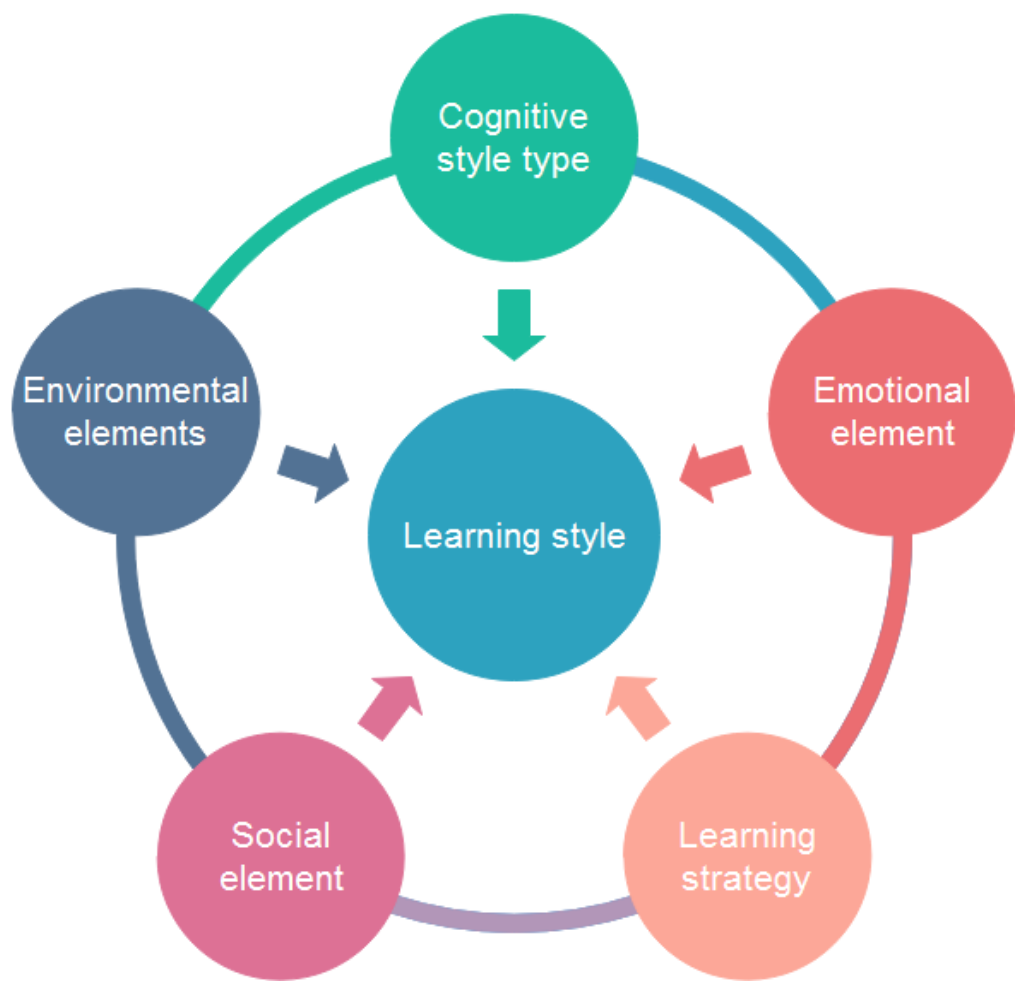

Fig. 5. Student learning style attribute

The differences in students' cognitive ability attributes are shown in Figure 6. Cognitive ability is mainly manifested in intelligence and cognitive premise behavior. Although intelligence is not the only factor that determines the effect of learning, in reality teaching, it is necessary to distinguish the intellectual level of students. Among the eight kinds of intelligence proposed by Gardner, the types of intelligence that individuals and individuals possess and excel at are different. In the teaching, it is necessary to distinguish the differences in the types of students' intelligence and teach them in accordance with their aptitude. Cognitive premise behavior refers to the basic knowledge that students have before they learn a course. Teachers should control the students' recent development areas and promote the maximum development of students. 


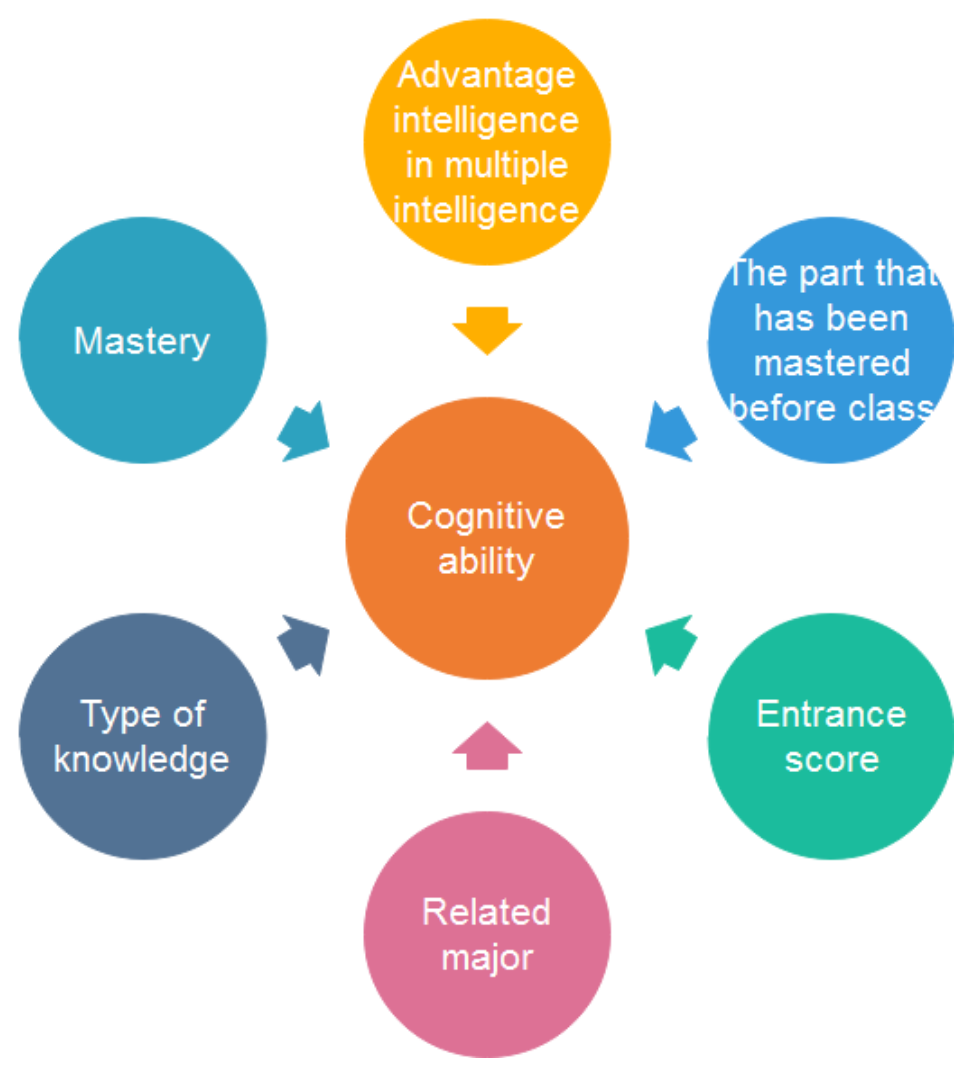

Fig. 6. Student cognitive ability

\section{Naive Bayesian Classification Algorithm}

\subsection{Bayesian theory}

The Naive Bayes classifier is an algorithm based on Bayesian theory in the classification algorithm set. Its advantage is that the algorithm is relatively simple, the estimated parameters are less, and the efficiency is relatively high, which can meet the speed requirement of large data volume training and query [11]. Its ideological basis is that when solving the category of the item to be classified, it is first assumed that the attributes of the item to be classified are independent, and then the prior probability of the object is solved, and the Bayesian formula is used to calculate the conditional probability that the object belongs to each category. The one with the largest value selected from the probability values is the category to which the item to be classified belongs.

Bayesian theory refers to calculating the probability of occurrence of another event based on the probability of an event that has occurred. The Bayesian theory is based on the following formula: 


$$
P(A \mid B)=\frac{P(B \mid A) P(A)}{P(B)}
$$

In the formula, $\mathrm{P}(\mathrm{A})$ and $\mathrm{P}(\mathrm{B})$ respectively represent the probability of occurrence of events $A$ and $B$. The value of conditional probability $P(B \mid A)$ is often easily obtained, and the conditional probability $\mathrm{P}(\mathrm{A} \mid \mathrm{B})$ is generally The result of the Bayesian formula solution is that the value can be obtained after some inference calculation.

\subsection{Naive hypothesis}

Bayesian theory adds the assumption that each feature is independent of each other. So we can divide the event into separate parts.

If the two events are independent, then there are:

$$
P(A B)=P(A) P(B)
$$

which is:

$$
P\left(\mathrm{y} \mid x_{1}, \cdots, x_{n}\right)=\frac{P\left(x_{1} \mid y\right) P\left(x_{2} \mid y\right) \cdots P\left(x_{n} \mid y\right)}{P\left(x_{1}\right) P\left(x_{2}\right) \cdots P\left(x_{n}\right)}
$$

Equivalent to

$$
P\left(\mathrm{y} \mid x_{1}, \cdots, x_{n}\right)=\frac{P(\mathrm{y}) \prod{ }_{i=1}^{n} P\left(x_{i} \mid y\right)}{P\left(x_{1}\right) P\left(x_{2}\right) \cdots P\left(x_{n}\right)}
$$

The denominator is constant related to the input data, so we can remove this one:

$$
P\left(\mathrm{y} \mid x_{1}, \cdots, x_{n}\right)_{\infty}=P(\mathrm{y}) \prod_{i=1}^{n} P\left(x_{i} \mid y\right)
$$

Now we need to build a classification model. We calculate the probability with all possible values of the known class variable $\mathrm{y}_{\mathrm{y}}$ and choose the output probability to be the largest result. Mathematical expressions can be written like this:

$$
\widehat{y}=\arg \max _{y} P(y) \prod_{i=1}^{n} P\left(x_{i} \mid y\right)
$$

$\mathrm{P}(\mathrm{y}) \mathrm{P}(\mathrm{y})$ is also called a class probability, and $\mathrm{P}\left(\mathrm{x}_{\mathrm{i}} \mid \mathrm{y}\right) \mathrm{P}\left(\mathrm{x}_{\mathrm{i}} \mid \mathrm{y}\right)$ is also called a conditional probability. 


\section{Constructing a Differential Classification Model}

\subsection{Classification basis}

The student classification method based on the naive Bayesian algorithm is based on the student's information as a sample set for constructing a naive Bayesian classifier. The students are classified according to the student attribute information, and the students in the same class are not pure. The results are judged as criteria, but combined with other attributes, and classified by [12]. The naive Bayesian classification algorithm is based on Bayes' theorem and the maximal posterior hypothesis, assuming that the attributes of a given entity are independent of each other and independent of each other. When calculating the joint probability, only the calculation will be performed. The posterior probabilities of each attribute value are multiplied, and the product is expressed as a joint probability. This eliminates the computational joint probability by eliminating a rather complicated computational process, which greatly improves its efficiency. The naive Bayesian classification algorithm is based on Bayes' theorem and uses probability to classify students. From the perspective of the content of the difference teaching theory, this paper selects 20 attributes from five dimensions to analyze the students. Differences, then by the statistical sample set of students classification and the number of students in each class, calculate the probability of the student category and the conditional probability of each attribute in each class, these data are prior probability, after the conclusion The probability of probing provides data support. Finally, the calculated prior probability is used to calculate the conditional probability that each student to be classified belongs to each class, that is, the posterior probability, and the maximum probability value of the category is selected, which is the category of the newborn. At the same time, the prior probability is recalculated and updated, which completes the process of classification.

\subsection{Classification process}

The theoretical research and practical teaching of differential teaching is based on the student's differential classification. The student classification method based on the naive Bayesian algorithm is based on the student's attribute value difference and the scientific difference classification is based on the sample. Using the naive Bayesian algorithm, the students realized the effect of classifying the classifiers once [13]. The method can effectively and scientifically realize the influence of the subjective factors in the classification of students in the class division of students, and avoid the single division of academic achievement as a standard, so as to avoid the situation that students do not understand. The blind grouping is convenient for the future to realize the difference teaching of the students after classification, which helps to improve the teaching effect of implementing the difference teaching. The entire classification process is roughly divided into two phases.

The first phase data collection: In the attribute information collection stage, it is necessary to combine the relevant theories of difference teaching to analyze which 
attributes are used to express student differences. Which attributes can be effectively and rationally classified by students, and the classification is truly realized, avoiding the single attribute as Classification criteria. Then based on the determined key attributes, the design questionnaire is sent, and the questionnaire is issued to the students; then the key information of the students is collected, the collected data is preprocessed, and the data format used in accordance with the method is processed. As shown in Figure 7.

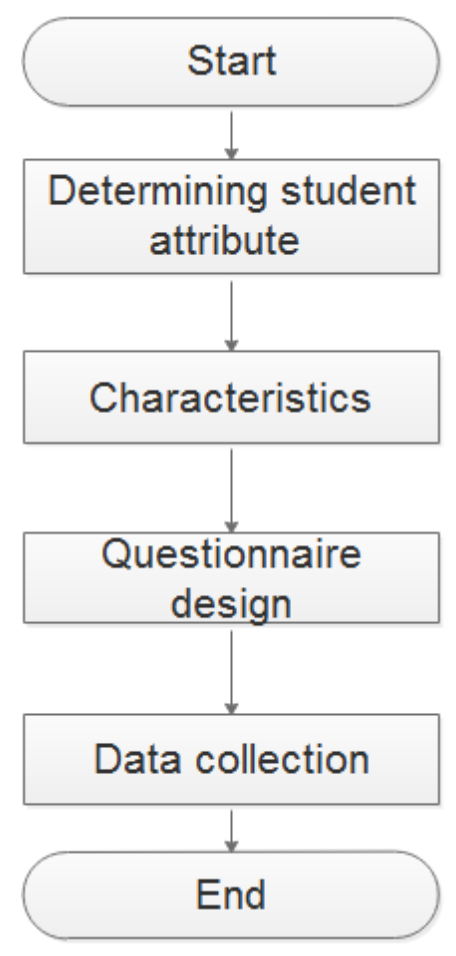

Fig. 7. Data collection flow chart

The second stage data processing: The second stage is the training stage, which mainly completes the construction of the classifier. Using the formulas (3-1), (3-2), the class probability and the conditional probability of each feature attribute value in the category are calculated respectively. Probability. In the classifier construction process, the input of this stage is the training sample data output in the preparation stage, and the output is the previous classifier. This stage is the core part of the whole classification method. The program will automatically update the conditional probability of each category's probability and feature attributes in each category, and record the calculation results to provide data support for the application stage. As shown in Figure 8 . 


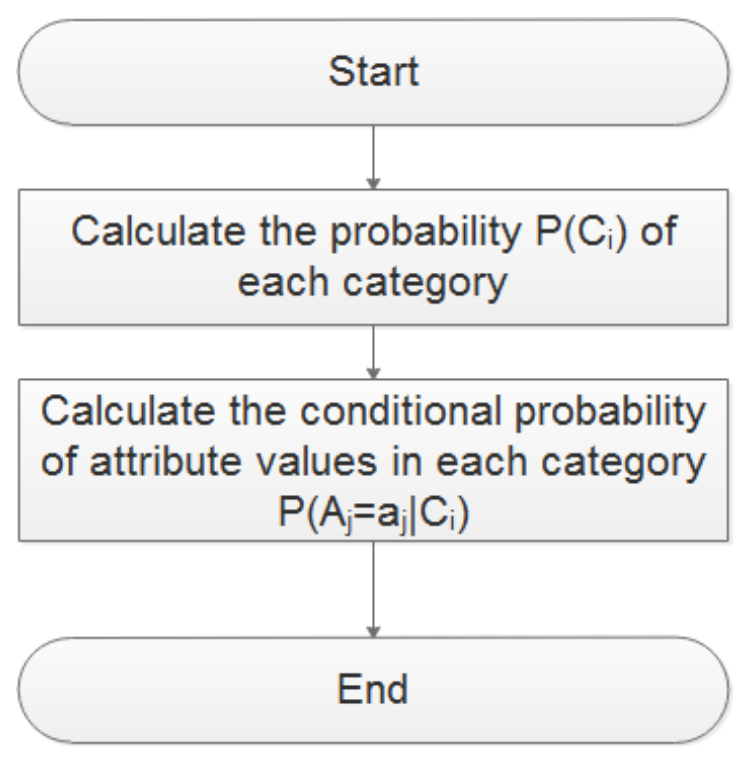

Fig. 8. Data processing flow chart

At this stage, formulas (3-3) _ (3-6) are used to calculate the conditional probability of the students to be classified under each student category, the conditional probability of the students to be classified as belonging to the category, and finally calculate the largest of the students to be classified. The category probability, that is, the category to which the student to be classified belongs, ends the classification of the student.

\section{$5 \quad$ Application in Practical Teaching}

\subsection{Research object}

A college electives two classes of 《financial accounting》, a total of 120 students are divided into 2 groups, A group experimental group, 60 students, B group control group, 60 students.

\subsection{Differential analysis}

The questionnaires were distributed to determine the cognitive status of the experimental group students. The questionnaires were designed according to 5 dimensions and 20 attributes, and then differentiated teaching according to the classification results of Naive Bayesian algorithm.

The control group was grouped according to the teacher's daily observations and the students' cognitive situation, and then differentiated teaching. 


\subsection{Classification process and data processing}

According to the questionnaire, the data classifier is constructed. After determining the classifier, the probability is calculated according to formula (3-1)-(3-4), and the classification is performed according to the probability value. The results of the course mastery and interest in learning are shown in Table 1 below:

Table 1. Difference classification part attribute analysis table

\begin{tabular}{|c|l|c|}
\hline \multicolumn{1}{|c|}{ Dimension } & \multicolumn{1}{|c|}{ Attributes } & Calculation results \\
\hline \multirow{4}{*}{ Course mastery } & Self-learned accounting knowledge & $15.7 \%$ \\
\cline { 2 - 3 } & Understand accounting course knowledge & $72.9 \%$ \\
\cline { 2 - 3 } & Don't know any accounting course knowledge & $11.4 \%$ \\
\hline \multirow{4}{*}{ Student interest } & like it very much & $42.6 \%$ \\
\cline { 2 - 3 } & like & $46.9 \%$ \\
\cline { 2 - 3 } & do not like & $10.5 \%$ \\
\hline
\end{tabular}

The experimental group students are classified according to the probability information collected by the collector. The classification is shown in Table 2:

Table 2. Classification table

\begin{tabular}{|l|c|c|}
\hline \multicolumn{1}{|c|}{ Category } & Number of people & Probability \\
\hline The first sort & 18 & $30 \%$ \\
\hline Second class & 20 & $33.3 \%$ \\
\hline Third category & 22 & $36.7 \%$ \\
\hline
\end{tabular}

\subsection{Result analysis}

The same teacher, using the same teaching materials, the experimental group used the differentiated teaching mode according to the Naïve Bayesian algorithm. The control group students used the traditional classification method to implement the differentiated teaching teaching mode. After the course study, the unified test paper test was conducted. The test results are as follows:

Table 3. Grade distribution table

\begin{tabular}{|l|c|c|c|c|c|}
\hline \multicolumn{1}{|c|}{ Group } & $\mathbf{2 0}$ & $\mathbf{8 0 - 9 0}$ & $\mathbf{7 0 - 8 0}$ & $\mathbf{6 0 - 7 0}$ & $\leqslant \mathbf{6 0}$ \\
\hline Control group & 3 & 17 & 22 & 14 & 4 \\
\hline Test group & 7 & 23 & 15 & 15 & 0 \\
\hline
\end{tabular}

\subsection{Verification analysis}

A college electives two classes of 《Cost accounting》, a total of 100 students are divided into 2 groups, A group experimental group, 50 students, B group control group, 50 students. 
The applied analysis of 《Cost accounting 》 is consistent with the above courses.

The same teacher, using the same teaching materials, the experimental group used the differentiated teaching mode according to the Naïve Bayesian algorithm. The control group students used the traditional classification method to implement the differentiated teaching mode. After the course study, the unified test paper test was conducted. The test results are as follows:

Table 4. Grade distribution table

\begin{tabular}{|l|c|c|c|c|c|}
\hline \multicolumn{1}{|c|}{ Group } & $\mathbf{2 0 0}$ & $\mathbf{8 0 - 9 0}$ & $\mathbf{7 0 - 8 0}$ & $\mathbf{6 0 - 7 0}$ & $\leqslant \mathbf{6 0}$ \\
\hline Control group & 4 & 13 & 19 & 11 & 3 \\
\hline Test group & 7 & 21 & 13 & 9 & 0 \\
\hline
\end{tabular}

Through the statistical analysis of the results, it is found that the classification of students is more accurately classified by the naive Bayesian algorithm. Through the final test results, it is found that this classification method is effective.

\section{Conclusion}

The naive Bayesian algorithm is applied to the class of students in a certain accounting department of the university to conduct classification experiments. Based on the characteristics of the students themselves, the multi-dimensional classification attributes are established, and the students are effectively classified. According to the test results, the differential classification of naive Bayesian algorithm is found. A good classification effect. The classification process is classified by mathematical probability calculation, which avoids the influence of human subjectivity on the classification result, so that the teacher can predict the student's needs in advance without contact with the students, and contribute to the difference teaching. Implementation. It also provides data support for theoretical research and practical teaching of differential teaching in the future.

\section{$7 \quad$ References}

[1] Wang Yiping. Some Thoughts on the Teaching Methods of Basic Accounting [J]. . Economic and Trade Practices. 2017(23)

[2] Wang Wei, Gao Kaili. A New Exploration of Experiential Teaching in Basic Accounting: Process Thinking [J]. Finance and Accounting News. 2014(28)

[3] Zhang Zaijun, Li Jianlong. Educational wisdom of famous Chinese and foreign educators "M]. Beijing: Petroleum Industry Press, 2011: 316, 312.

[4] [4] Leslie Lauder is waiting, Liu Wei translation. Differentiated teaching exploration: literature, mathematics and science "M]. Shanghai: East China Normal University Press, 2015.

[5] Jiang Zhi, Hua Guodong. The essence of "difference teaching" [[J]. China Education Journal, 2004, 04: 54-57. 
[6] Hua Guodong. Difference Teaching Theory "M]. Beijing: Educational Science Press, 2001.

[7] Hua Guodong. Difference Teaching Theory [Ml. Beijing: Education Science Press, 2007: 30-36, 16, 23-30.

[8] US) Heacox (D.), Yang Xijie translation. Difference teaching helps each student to succeed "M]. Beijing: China Light Industry Press, 2004::31, 37-79, 3, 51-79.

[9] Greg Gregory waits, translated by Zhao Liqin. Differentiated teaching "M]. Shanghai: East China Normal University Press, 2015.

[10] Song Chunna. Research on the Teaching Design of "Computer Foundation" Based on the Difference Teaching Thought "D]. Baoding: Hebei University, 2010.

[11] Research and application of Oman Naïve Bayesian classification algorithm [D]. Dalian: Dalian University of Technology, 2014.

[12] Dong Lei, Zhao Yumu, Guo Jihong. Discussion on Middle School Physics Teaching Strategies Based on Student Differences[J]. Modern Education Science, 2011, 12: 145146.

[13] Guo Xukun, Fan Bingbing A distributed parallel implementation of a naive Bayesian text classification algorithm[J]. Computer Applications and Software, 2016, 33(11): 240-243+296.

[14] Jiang Zhi, Hua Guodong. The essence of "differential teaching" [[J]. China Education Journal, 2004, 04: 54-57.

[15] Ren Shaobo. Research on the development of professional accounting courses in higher vocational schools fDl. Zhejiang University of Technology.

\section{Author}

Xiuying Ou, female, teacher of School of Management of WENZHOU BUSINESS COLLEGE, Lecturer, Master, lectured on courses like 《Basic Accounting $\rangle$, 《Bank Accounting》, 《Comprehensive Experiment of Enterprise Accounting $\rangle$, and 《Cost Accounting》. I am mainly engaged in the teaching and research of accounting theory and practice. In recent years, I have published many articles in the core journals. Participated in compiling textbooks such as 《Basic Accounting》; Participate in the construction of several educational reform projects. Email id: xgyue@,foxmail.com

Article submitted 2019-02-04. Resubmitted 2019-03-06. Final acceptance 2019-03-06. Final version published as submitted by the authors. 